\title{
Enhanced rice salinity tolerance via CRISPR/ Cas9-targeted mutagenesis of the OsRR22 gene
}

\author{
Anning Zhang • Yi Liu • Feiming Wang • Tianfei Li • Zhihao Chen • Deyan Kong • \\ Junguo Bi • Fenyun Zhang • Xingxing Luo • Jiahong Wang • Jinjuan Tang • Xinqiao Yu • \\ Guolan Liu • Lijun Luo
}

Received: 2 July 2018 / Accepted: 19 February 2019/Published online: 9 March 2019

(C) The Author(s) 2019

\begin{abstract}
Salinity is one of the most important abiotic stress affecting the world rice production. The cultivation of salinity-tolerant cultivars is the most costeffective and environmentally friendly approach for salinity control. In recent years, CRISPR/Cas9 systems have been widely used for target-site genome editing; however, their application for the improvement of elite rice cultivars has rarely been reported. Here, we report the improvement of the rice salinity tolerance by engineering a Cas9-OsRR22-gRNA expressing vector, targeting the $O S R R 22$ gene in rice. Nine mutant plants were identified from $14 \mathrm{~T}_{0}$ transgenic plants. Sequencing showed that these plants had six mutation types at the target site, all of which were successfully transmitted to the next generations. Mutant plants without transferred DNA (T-DNA) were obtained via segregation in the T1 generations. Two T2 homozygous mutant lines
\end{abstract}

Anning Zhang and Yi Liu contributed equally to this work.

Electronic supplementary material The online version of this article (https://doi.org/10.1007/s11032-019-0954-y) contains supplementary material, which is available to authorized users.

A. Zhang $\cdot$ Y. Liu $\cdot$ L. Luo

Huazhong Agricultural University, Wuhan 430070, People's Republic of China

A. Zhang $\cdot$ Y. Liu $\cdot$ F. Wang $\cdot$ T. Li $\cdot$ Z. Chen $\cdot$ D. Kong $\cdot$ $\mathrm{J}$. Bi $\cdot$ F. Zhang $\cdot \mathrm{X}$. Luo $\cdot \mathrm{J}$. Wang $\cdot \mathrm{J}$. Tang $\cdot \mathrm{X}$. Yu $\cdot$

G. Liu $(\bowtie) \cdot$ L. Luo $(\bowtie)$

Shanghai Agrobiological Gene Center, Shanghai 201106, People's Republic of China

e-mail: guolanliu@126.com

e-mail: lijun@sagc.org.cn were further examined for their salinity tolerance and agronomic traits. The results showed that, at the seedling stage, the salinity tolerance of T2 homozygous mutant lines was significantly enhanced compared to wild-type plants. Furthermore, no significantly different agronomic traits were found between T2 homozygous mutant lines and wild-type plants. Our results indicate CRISPR/ Cas9 as a useful approach to enhance the salinity tolerance of rice.

Keywords CRISPR/Cas9 - OsRR22 · Salinity tolerance $\cdot$ Genetic engineering $\cdot$ Abiotic stress

\section{Introduction}

The global crop production needs to double by 2050 to match the demands of the rapidly increasing population, changing diet, and increasing biofuel consumption (Ray et al. 2013). However, abiotic stress, which includes drought, salinity, low temperature, heat, flooding, and oxidative stress, severely limits the feasible yield increase, or even reduces crop production in large areas (Mahajan and Tuteja 2005). Among these abiotic stress, salinity poses one of the major threats to crop production since most crop plants cannot grow under a high concentration of salt (Munns and Tester 2008). Furthermore, soil salinity is hard to remove, which will cause a continuous decrease in crop production for many years. Over 400 million hectares of land throughout the world have been affected by salinity 
(http://www.plantstress.com/Articles/index.asp). Moreover, land affected by salt stress is arising due to various factors such as climate change, sea-level increases, and tsunamis (Kumar et al. 2013). Consequently, salinity remains a severe threat to the food supply.

Rice (Oryza sativa L.) is one of the most important food crops and forms the main staple food for more than half of the world's population. Since rice is a species originally grown in swamps and freshwater marshes, it is particularly sensitive to salt stress and rated as a particularly salt-sensitive crop (Dionisio-Sese and Tobita 1998; Kumar et al. 2013). Salinity is one of the major obstacles for rice production especially at the seedling stage (Lutts et al. 1995). Researching of rice salt tolerance is becoming increasingly urgent and improving the salt tolerance of rice has become an important breeding goal. Numerous salt tolerance quantitative trait loci were identified and few of them had been transferred into popular rice varieties via markerassisted selection (MAS) (Lang et al. 2011; Bimpong et al. 2016; Jing and Zhang. 2017). During the past two decades, many salt-related genes (SKC1, DST, OsRR22, OSHAL3, P5CS, SNAC2, and OSNAP) have been successfully cloned (Ren et al. 2005; Hu et al. 2008; Huang et al. 2009; Sun et al. 2009; Karthikeyan et al. 2011; Chen et al. 2014; Takagi et al. 2015). Among them, the OsRR22 gene encodes a 696-amino acid B-type response regulator transcription factor that is involved in both cytokinin signal transduction and metabolism; its loss of function has been reported to significantly increase salt tolerance (Takagi et al. 2015).

The CRISPR/Cas9 system is an accurate, convenient, and efficient genome-editing method developed during recent years (Shan et al. 2013). At present, the CRISPR/Cas9 system has been widely used for genome editing in major crops such as wheat (Wang et al. 2014; Liang et al. 2017), maize (Svitashev et al. 2016; Zhu et al. 2016), and sorghum (Li et al. 2015; Cai et al. 2015). In rice, using CRISPR/Cas9 technology, many genes (OSPDS, OsERF922, OsHAK1, Badh2, and TMS5) have been knocked out and the expected phenotype was obtained (Zhang et al. 2014; Wang et al. 2016; Zhou et al. 2016; Nieves-Cordones et al. 2017; Shao et al. 2017). This system provides a new method for rice breeding. This study first reports the improvement of salinity tolerance via CRISPR/Cas9-targeted mutagenesis of the transcription factor OsRR22.

\section{Materials and methods}

Plant growth conditions

The elite japonica rice cultivar WPB106 was bred from 'Huhan9/Huxiangjing//Huhan3/Huhan11' in our laboratory. All transgenic plants and WPB106 (wild type, WT) were grown in the greenhouse at $28-35^{\circ} \mathrm{C}$, in Shanghai, or in fields at the station of the Shanghai Academy of Agricultural Sciences under normal growth conditions. For salinity stress at the seedling stage, seedlings of rice were cultivated in normal nutrient solution for 5 days after germination on a 96-well plate (Xia et al. 2017). They were placed in a growth chamber ( $14 \mathrm{~h}$ of daytime at $30^{\circ} \mathrm{C}$ and $10 \mathrm{~h}$ at night at $20^{\circ} \mathrm{C}$ with $70 \%$ relative humidity).

Vector construction

The Cas9 plant expression vector (pYLCRISPR/ Cas9Pubi-H) and the sgRNA expression vector (pYLgRNA) were provided by Prof. Yao-Guang Liu of the South China Agricultural University. The Cas9-OsRR22-gRNA expressing vector was constructed following previously described protocol (Ma et al. 2015a). Briefly, according to the design principles of the target sequences of the CRISPR/ Cas 9 system, 19 to 20 bases upstream of the protospacer adjacent motif (PAM) were selected as candidate target sequence (Fig. 1 a). A BLAST search (http://blast.ncbi.nlm.nih.gov/Blast.cgi) of the target sequences (including PAM) against the rice genome was conducted to confirm their targeting specificity in the genome. The target sequence has a difference of at least two bases compared with similar non-target sequences within the PAM or PAM-proximal region. The gRNA expression cassette was synthesized via overlapping PCR. The target-specific sequence of gRNA (target OsRR22) was put at the 5'-end of the primers RR22gRT+/RR22-OsU6aT-. Two PCR reactions were performed, using the plasmid pYLgRNA-OsU6a/LacZ as template. The first PCR was performed using the primer set U-F/RR22-OsU6aT-, and the second one used the primer set RR22-gRT+/gR-R (Table 1). The products of PCR 1 and 2 were used as templates for the third PCR reaction with the primer set UGAL/Pgs-GAR to generate the full-length gRNA fragment (Table 1). Subsequently, amplicons 
(a)

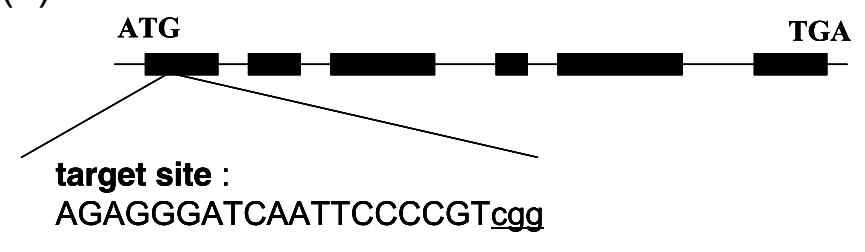

(b)

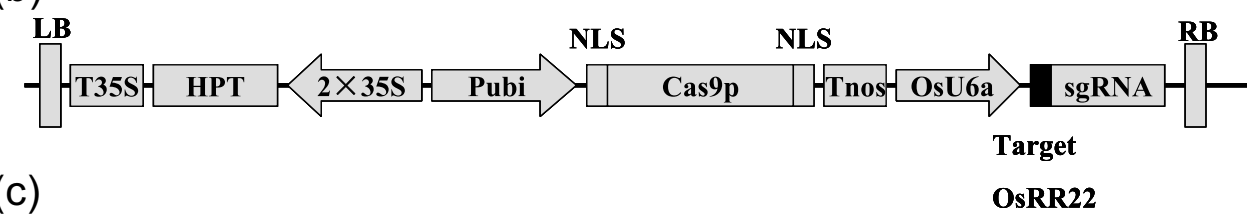

\begin{tabular}{|c|c|c|}
\hline Wild type & tggggagggAGAGGGATCAATTCCCCGTcggcatgcg & \\
\hline \multicolumn{3}{|l|}{ Homozygote } \\
\hline $\mathbf{r r}-2$ & tggggagggAGAGGGATCAATTCCCCCGTcggcatgcg & i1 \\
\hline rr-7 & tggggagggAGAGGGATCAATTCCCACGTcggcatgcg & i1 \\
\hline \multicolumn{3}{|l|}{ Heterozygote } \\
\hline \multirow[t]{2}{*}{$\mathbf{r r}-4$} & tggggagggAGAGGGATCAATTCССТССТcggcatgeg & $\mathrm{i} 1 / \mathrm{s} 1$ \\
\hline & tggggagggAGAGGGATCAATTCCCCGTcggcatgcg & WT \\
\hline \multirow[t]{2}{*}{ rr-10 } & tggggagggAGAGGGATCAATTCCC---cggcatgeg & d3 \\
\hline & tggggagggAGAGGGATCAATTCCCCGTcggcatgcg & WT \\
\hline \multicolumn{3}{|l|}{ Bi-allelic } \\
\hline \multirow[t]{2}{*}{ rr-1 } & tggggagggAGAGGGATCAATTCCC-CGTçggcatgcg & d1 \\
\hline & tggggagggAGAGGGATCAATTCCCGCGTcggcatgcg & i1 \\
\hline \multirow[t]{2}{*}{ rr-6 } & tggggagggAGAGGGATCAATTCCC-TCcggcatgcg & $\mathrm{d} 1 / \mathrm{s} 2$ \\
\hline & tggggagggAGAGGGATCAATTCCC-GCCGGCT $\underline{\text { cggcatgcg }}$ & $\mathrm{d} 1 / \mathrm{i} 5$ \\
\hline \multirow[t]{2}{*}{ rr-9 } & tggggagggAGAGGGATCAATTCCСTCGTcggcatgeg & i1 \\
\hline & tggggagggAGAGGGATCAATTCCC-GCCTCCGTcggcatgeg & d1/i6 \\
\hline \multirow[t]{2}{*}{ rr-11 } & tggggagggAGAGGGATC--T-CCCCGCcggcatgcg & $\mathrm{d} 3 / \mathrm{s} 2$ \\
\hline & tggggagggAGAGGGATCAATTCCCTCGTcggcatgcg & i1 \\
\hline \multirow[t]{2}{*}{ rr-12 } & tggggagggAGAGGGATCAATTCССТССTcggcatgeg & i1 \\
\hline & tggggagggAGAGGGATCAATTCССССТcggcatgcg & s1 \\
\hline
\end{tabular}

Fig. 1 CRISPR/Cas9-induced OsRR22 gene modification in rice. a Schematic of the $O s R R 22$ gene structure and target site. Exons and introns are indicated with black rectangles and black lines, respectively. Both the translation initiation codon (ATG) and the termination codon (TGA) are shown. The target site nucleotides are shown in capital letters and the protospacer adjacent motif (PAM) site is underlined. b Schematic presentation of the T-DNA structure in the CRISPR/Cas9-mediated genome editing construct. The expression of Cas9 is driven by the maize ubiquitin promoter (Pubi); the expression of the sgRNA scaffold is driven by the rice U6a small nuclear RNA promoter (OsU6a); the expression of hygromycin (HPT) is driven by two CaMV35S promoters $(2 \times$

containing OsRR22-gRNA with different BsaI-cutting sites were cloned into the Cas9 plant expression vector pYLCRISPR/Cas9Pubi-H at the BsaI site,
35S). Abbreviations: NLS, nuclear localization signal; Tnos, gene terminator; $\mathrm{LB}$ and $\mathrm{RB}$, left border and right border, respectively. $\mathrm{c}$ Nucleotide sequences at the target site in the nine $T_{0}$ mutant rice plants. The recovered mutated alleles are shown below the wildtype sequence. The target site nucleotides are indicated with black capital letters. The PAM site is underlined. The red dashes indicate deleted nucleotides. The red capital letters indicate inserted or substituted nucleotides. The numbers on the right indicate the type of mutation and the number of nucleotides involved. "i," "d," and "s" indicate insertion, deletion, and substitution of the indicated number of nucleotides, respectively; "WT" indicates wild-type

using the pEASY-Uni Seamless Cloning and Assembly Kit (TransGen Biotech, Beijing, China). The resultant construct Cas9-OsRR22-gRNA contained 
Table 1 Primers used in this study

\begin{tabular}{llc}
\hline Primer name & Primer sequence $\left(5^{\prime}-3^{\prime}\right)$ & Purpose \\
\hline RR22-gRT+ & AGAGGGATCAATTCCCCGTgtttagagctagaaat & Vector construct \\
RR22-OsU6aT- & ACGGGGAATTGATCCCTCTCggcagccaagccagca & Vector construct \\
U-F & CTCCGTTTTACCTGTGGAATCG & Vector construct \\
gR-R & CGGAGGAAAATTCCATCCAC & Vector construct \\
U-GAL & ACCGGTAAGGCGCGCCGTAGTGCTCGACTAGTATGGAATCGGCAGCAAAGG & Vector construct \\
Pgs-GAR & TAGCTCGAGAGGCGCGCCAATGATACCGACGCGTATCCATCCACTCCAAGCTCTTG & Vector construct \\
RR22-S-F & CTTGGGATTGCTCTGTTTCT & Target site sequencing \\
RR22-S-R & GTAATAGCCTGGTTGGTTGAT & Target site sequencing \\
HPT-F & GCTCCATACAAGCCAACCACG & Transgenic analysis \\
HPT-R & CCTGCCTGAAACCGAACTGC & Transgenic analysis \\
CAS9-F & CGAGACGAACGGTGAGACTGGTG & Transgenic analysis \\
CAS9-R & GGTGCTTGTTGTAGGCGGAGAGG & Transgenic analysis \\
\hline
\end{tabular}

a Cas9p expression cassette (Pubi::NLS::Cas9 p::NLS::Tnos) and a hygromycin resistance cassette (2×P35S::HPT::T35STnos) (Fig. 1 b).

\section{Agrobacterium-mediated rice transformation}

The Cas9-OsRR22-gRNA expressing binary vector was introduced into the japonica rice WPB106 cultivar via the Agrobacterium-mediated transformation method described by Nishimura et al. (2006). Hygromycincontaining medium was used to select hygromycinresistant calli, and then, vigorously growing calli were transferred to regeneration media to generate green plants.

Identification of mutant transgenic plants

To determine the mutation at the target site, genomic DNA from the leaves of transgenic plants was extracted using a DNA Quick Plant System (TransGen Biotech, Beijing, China). Genomic DNA (50 ng) was used as template to perform PCR amplification using PCR Mastermix (TIANGEN, Beijing, China). PCR was performed to amplify the genomic region containing the CRISPR/Cas9 target site, using specific primer pairs that surround the designed target site (Table 1). The PCR products were directly sequenced or cloned into the pEASY-Blunt vector (TransGen Biotech, Beijing, China) and sequenced using the Sanger method. Mutations were identified by comparing the sequences of transgenic plants with those of WT plants. Mutations containing normal sequencing chromatograms were considered as homozygote mutations. Mutations containing superimposed sequencing chromatograms were considered heterozygous or bi-allelic mutations, which were decoded via degenerate sequence decoding (Ma et al. 2015b).

To identify T-DNA-free plants from $\mathrm{T}_{1}$, the plants were analyzed via PCR using HPT-specific and Cas9specific primers (Table 1) in combination with agarose gel electrophoresis. The $\mathrm{pYLCRISPR/Cas9Pubi-H} \mathrm{plas-}$ mids and the $T_{0}$ transgenic plants were selected as positive controls and WPB106 DNA and $\mathrm{H}_{2} \mathrm{O}$ were used as negative controls. HPT- and $\operatorname{Cas} 9$-negative plants were considered as T-DNA-free plants.

Greenhouse trials for salinity tolerance

To evaluate the salinity tolerance of plants at the seedling stage, a salt stress test was performed according to the method published by Takagi et al. (2015). We compared the salinity tolerance of 2-week-old WT and homozygous mutant plants in the greenhouse of the Shanghai Agrobiological Gene Center, Shanghai. Briefly, 2-week-old plants were treated with fresh groundwater and concentrations of $0.75 \% \mathrm{NaCl}$ solution $(\mathrm{pH}=7)$, respectively. After 2 weeks of treatment, the salinity tolerance was determined via plant height and shoot fresh and dry weights of 10 plants per line. Each line was replicated three times. 
Evaluation of major agronomic traits under field conditions

To evaluate the agronomic traits under normal field conditions, both WT and mutant plants were planted in a four-row plot with seven plants per row, applying $20 \times$ $15 \mathrm{~cm}$ spacing in Shanghai, China, during the summer of 2017. In these field trials, 4-week-old plants (or older) were transplanted and field management was conducted according to local conventional methods. The agronomic traits were measured according to the SES (IRRI 2002). Five plants in the middle row of each line were sampled for the following agronomic traits: days to $50 \%$ flowering, plant height, no. of tillers, no. of grains per panicle, spikelet fertility, 1000-seed weight, and yield per plant.

\section{Results}

\section{CRISPR/Cas9 design}

To design a mutation specifically targeting the OsRR22 gene in rice, a 20-bp nucleotide sequence in the first exon of OsRR22 (GenBank Accession No BR000251.1) was chosen as the target site (Fig. 1 a). The binary plasmid Cas9-OsRR22gRNA (Fig. 1 b) was constructed based on the CRISPR/Cas9 vector previously described by $\mathrm{Ma}$ et al. (2015a). The vector was used to transform the rice variety WPB106 via Agrobacterium-mediated transformation. Using site-specific PCR and Sanger sequencing, a total of nine WPB106 mutants were recovered from $14 \mathrm{~T}_{0}$ hygromycinresistant transgenic WPB106 plants $(64.3 \%)$. These nine mutants were detected and then subjected to zygosity analysis by cloning PCR products into the $\mathrm{T}$ vector for DNA sequencing. Sequence analyses detected two homozygous mutations, two heterozygous mutations, and five bi-allelic mutations. Based on allele mutation types, $44.4 \%$ of the mutations were nucleotide insertions, $11.1 \%$ of the mutations were nucleotide deletions, and $5.6 \%$ of the mutations were nucleotide substitutions; $11.1 \%$, $5.6 \%$, and $11.1 \%$ of the mutations were simultaneous nucleotide insertions and deletions, insertions and substitutions, and deletions and substitutions, respectively (Table 2).
Transmission of CRISPR/Cas9-induced mutations to the $\mathrm{T}_{1}$ generation

To further understand the inheritance of the mutation, two homozygous (rr-2, rr-7), one heterozygous (rr-10), and one bi-allelic (rr-1) $\mathrm{T}_{0}$ mutant plants (Fig. 1 c) were self-pollinated, and their progenies were genotyped at the target site. We randomly selected nine to $23 \mathrm{~T}_{1}$ progenies derived from each $\mathrm{T}_{0}$ plant for genotyping analysis (Table 3). As expected, all of these $T_{0}$ putative homozygotes and their offspring had identical genotypes (rr-2 and rr-7), suggesting stable transmittance of the mutations in these homozygous mutant lines to the next generation. Bi-allelic mutations in $\mathrm{T}_{0}$ plants were apparently transmitted to the $T_{1}$ generation following the Mendelian genetic law, indicating that the targeted mutations in $T_{0}$ plants were inherited normally. For example, the bi-allelic $\mathrm{T}_{0}$ mutant plant rr-1 harbors two mutations (a 1-bp deletion (d1) and a 1-bp insertion (i1)); its $\mathrm{T}_{1}$ progenies segregated in a ratio of 5 (d1):8 (d1/i1):4 (i1), which is consistent with the predicted Mendelian segregation $\left(\chi^{2}=0.176\right.$ $\left.<\chi_{0.05,2}^{2}=5.99\right)($ Table 3$)$. For the $\mathrm{T}_{1}$ generation of heterozygous (rr-10), several new mutations (6 $(\mathrm{d} 1 / \mathrm{i} 1))$ were generated. In combination, these results clearly demonstrated that CRISPR/Cas9induced gene mutations could be stably transmitted to subsequent generations.

\section{Selection of T-DNA-free mutant rice lines}

To obtain rice lines harboring the desired OsRR22 mutations without T-DNA of the construct Cas9OsRR22, we conducted PCR amplification using the primer sets designed to amplify Cas9 and HPT sequences (Table 1). The absence of transgenes was determined via negative PCR results of both Cas9 and HPT. T-DNA-free plants were found among most $\mathrm{T}_{1}$ plants, with the proportion ranging from 20.0 to $33.3 \%$ (Table 3 ). These results indicated that T-DNA-free homozygous mutants could be acquired via segregation populations. We isolated two TDNA-free homozygous mutant lines (rr-2-1 and rr7-4) in the $T_{1}$ generation to produce the $T_{2}$ population to identify the salinity-tolerant phenotypes, designated as WPB106-cas-1 and WPB106-cas-2. 
Table 2 Ratios of mutant genotype and mutation type at the target site in $\mathrm{T}_{0}$ mutant plants

\begin{tabular}{|c|c|c|c|c|c|c|c|c|}
\hline \multicolumn{3}{|c|}{ Mutant genotype ratios $(\%)^{\mathrm{a}}$} & \multicolumn{6}{|c|}{ Mutation type ratios $(\%)^{b}$} \\
\hline Bi-allele & Homozygote & Heterozygote & Deletion & Insertion & Substitution & $\begin{array}{l}\text { Insertion and } \\
\text { deletion }\end{array}$ & $\begin{array}{l}\text { Insertion and } \\
\text { substitution }\end{array}$ & $\begin{array}{l}\text { Deletion and } \\
\text { substitution }\end{array}$ \\
\hline $55.6(5 / 9)$ & $22.2(2 / 9)$ & $22.2(2 / 9)$ & $11.1(2 / 18)$ & $44.4(8 / 18)$ & $5.6(1 / 18)$ & $11.1(2 / 18)$ & $5.6(1 / 18)$ & $11.1(2 / 18)$ \\
\hline
\end{tabular}

${ }^{a}$ Based on the number of each mutant genotype out of the total number of all mutant genotypes at the target site

${ }^{\mathrm{b}}$ Based on the number of each allele mutation type out of the total number of all allele mutation types at the target site

Salinity tolerance was enhanced in OsRR22-induced mutations

To evaluate the salinity-tolerant phenotype of the obtained rice mutants, two homozygous mutant $\mathrm{T} 2$ lines (WPB106-cas-1 and WPB106-cas-2) with different allelic mutations and WT plants were treated with fresh groundwater and a concentration of $0.75 \% \mathrm{NaCl}$ nutrition solution at the 2-week-old stage. Compared to WT, two mutant lines grew better than WT under this condition (Fig. 2 a). As measured after 2 weeks of treatment, the shoot fresh weight of WT was reduced by $50.3 \%$, while WPB106-cas-1 and WPB106-cas-2 showed only $10.1 \%$ and $2.1 \%$ reduction in shoot fresh weight, compared to plants that were grown with fresh groundwater. Similarly, the shoot dry weight of WT had been reduced by $42.6 \%$, whereas WPB106-cas-1 and WPB106-cas-2 showed only decreases of $12.9 \%$ and $12.3 \%$. The $0.75 \%$ $\mathrm{NaCl}$ treatment also caused decreases of $31.8 \%, 20.3 \%$, and $17.8 \%$ in plant height of WT, WPB106-cas-1, and WPB106-cas-2, respectively (Fig. 2 b and Table S1). The significant difference analysis of the shoot fresh weight, shoot dry weight, and plant height indicated that two mutant lines were significantly different from WT plants. These results implicitly indicate that CRISPR/
Cas9-induced mutations in the OsRR22 gene enhanced the tolerance to salinity.

The main agronomic traits were not altered in rice mutants

To survey whether mutations in the OsRR22 gene affect other agronomic traits, we characterized two homozygous $\mathrm{T}_{2}$ mutant lines by measuring their plant height, days to $50 \%$ flowering, no. of tillers per plant, no. of grains per panicle, spikelet fertility, 1000-seed weight, and yield per plant under normal field conditions. Student's $t$ test showed that none of the $\mathrm{T}_{2}$ mutant lines was significantly different from WT plants under normal growth conditions (Table 4). These results showed that CRISPR/Cas9-induced mutations in the OsRR22 gene did not significantly influence agronomic traits under normal field conditions.

\section{Discussion}

CRISPR/Cas9 is a new genome-editing technique, which is highly specific and efficient. So far, the CRISPR/Cas9 technology has been widely used to

Table 3 CRISPR/Cas9-induced mutations in $O S R R 22$ and their transmission to the $\mathrm{T}_{1}$ generation

\begin{tabular}{|c|c|c|c|c|c|c|}
\hline \multirow[t]{2}{*}{$\mathrm{T}_{0}$ plant } & \multirow[t]{2}{*}{ Genotype } & \multirow[t]{2}{*}{ Mutation type } & \multirow{2}{*}{$\begin{array}{l}\text { Number of } \mathrm{T}_{1} \\
\text { plants tested }\end{array}$} & \multicolumn{2}{|c|}{ Mutation transmission in the $T_{1}$ generation } & \multirow{2}{*}{$\begin{array}{l}\text { No. of T-DNA-free } \\
\text { plants }\end{array}$} \\
\hline & & & & Targeted mutations & $\chi^{2}(1: 2: 1)$ & \\
\hline $\mathrm{rr}-2$ & Homozygote & i1 & 10 & 10 (i1) & ND & 2 \\
\hline $\mathrm{rr}-7$ & Homozygote & i1 & 9 & 9 (i1) & ND & 3 \\
\hline $\mathrm{rr}-10$ & Heterozygote & $\mathrm{d} 3$ & 23 & 5 (d3), 8 (d3/wt), 4 (wt), 6 (d1/i1) & ND & 0 \\
\hline $\mathrm{rr}-1$ & Bi-allelic & $\mathrm{d} 1 / \mathrm{i} 1$ & 17 & $5(\mathrm{~d} 1), 8(\mathrm{~d} 1 / \mathrm{i} 1), 4(\mathrm{i} 1)$ & $0.176(P>0.05)$ & 4 \\
\hline
\end{tabular}

"i" and " $\mathrm{d}$ " indicate insertion and deletion of the indicated number of nucleotides, respectively; " $\mathrm{d} / \mathrm{i}$ " indicates the simultaneous deletion and insertion of the indicated number of nucleotides. The numbers on the right indicate the type of mutation and the number of nucleotides involved

$W T$, wild type; $N D$, not detected 


\section{(a)}

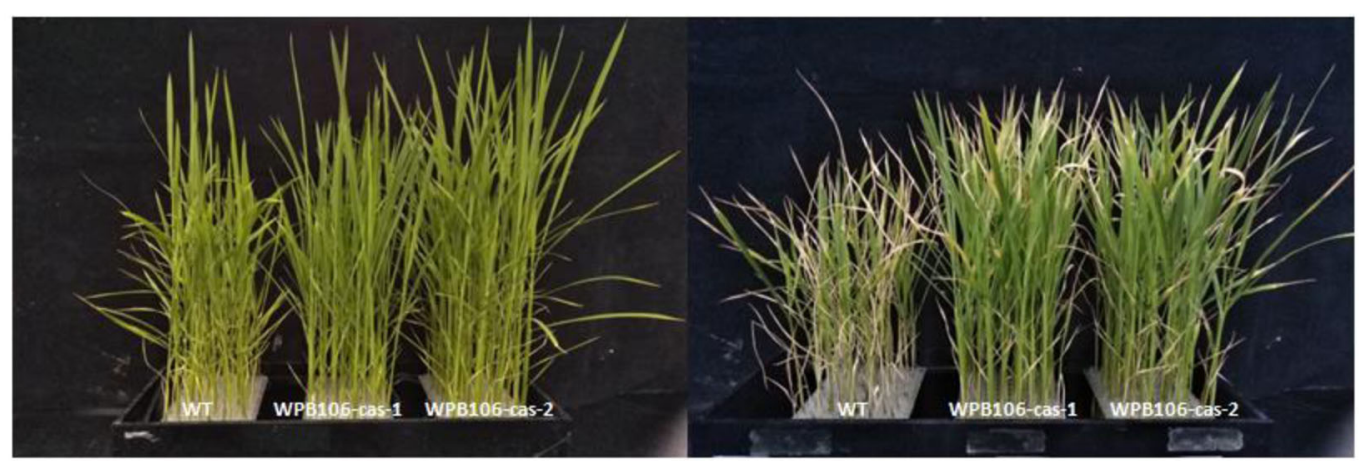

(b)

0

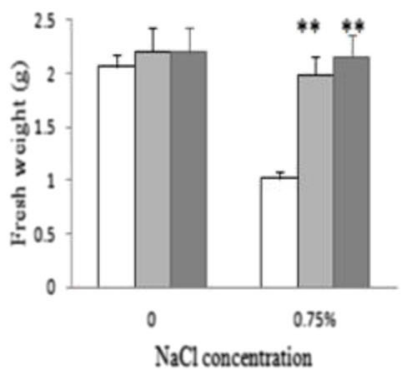

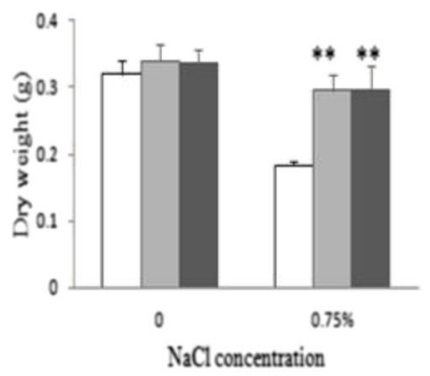

$0.75 \%$

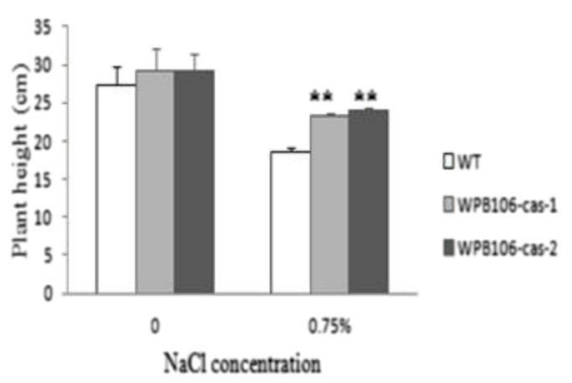

Fig. 2 Identification of salinity tolerance in homozygous mutant rice lines. a Phenotypes of 4-week-old WT, WPB106-cas-1, and WPB106-cas-2 plants grown with underground fresh water and subjected to a concentration of $0.75 \% \mathrm{NaCl}$. Two-week-old plants were treated with concentrations of $0.75 \% \mathrm{NaCl}$. Then, phenotypic evaluation was conducted 14 days after treatment. b A

improve major crops, such as rape, corn, rice, and soybean (Bortesi and Fischer 2015). However, few studies reported the direct genome editing of elite rice cultivars with the CRISPR/Cas9 technology. The ERF transcription factor gene OsERF922 was mutated by CRISPR/ Cas9 to enhance the blast resistance of the rice variety Kuiku131 with normal phenotypes (Wang et al. 2016). Knockout of the thermo-sensitive genic male-sterile (TGMS) gene tms 5 of 11 fertile elite cultivars produced TGMS lines with good agronomic characteristics (Zhou et al. 2016). WPB106, a water-saving and droughtresistant elite japonica cultivar (Luo 2010), has the advantages of drought resistance, early maturity, and

comparison of shoot fresh weight, shoot dry weight, and plant height between WT, WPB106-cas-1, and WPB106-cas-2 plants is shown in a. Values of shoot fresh weight and shoot dry weight represent weight of 10 plants per treatment. Mean values and standard deviations are shown. Asterisks indicate significant differences to WT $(* * P<0.01)$

cooking quality; however, it is very sensitive to salinity. To quickly improve its salt tolerance, we applied the CRISPR/Cas9 technology. In this study, we used the Cas9-OsRR22-gRNA expressing vector to knockout OsRR 22 and achieved $64.3 \%$ mutant plants in $\mathrm{T}_{0}$ transgenic plants. We obtained two homozygous mutant lines that harbor mutagenesis in OsRR22 without exogenous T-DNA. The evaluation of the salinity tolerance at the seedling stage showed that the salinity tolerance of $T_{2}$ homozygous mutant lines was significantly enhanced compared to that of WT plants. Furthermore, the result of field trials showed no significant difference between $\mathrm{T}_{2}$ homozygous mutant lines and WT plants in the main

Table 4 Agronomic traits of homozygous $\mathrm{T}_{2}$ mutant lines

\begin{tabular}{llllllll}
\hline Lines & $\begin{array}{l}\text { Days to 50\% } \\
\text { flowering }\end{array}$ & $\begin{array}{l}\text { Plant height } \\
(\mathrm{cm})\end{array}$ & $\begin{array}{l}\text { No. of tillers } \\
\text { per plant }\end{array}$ & $\begin{array}{l}\text { No. of grains } \\
\text { per panicle }\end{array}$ & $\begin{array}{l}\text { Spikelet } \\
\text { fertility }(\%)\end{array}$ & $\begin{array}{l}\text { 1000-seed } \\
\text { weight }(\mathrm{g})\end{array}$ & $\begin{array}{l}\text { Yield per } \\
\text { plant }(\mathrm{g})\end{array}$ \\
\hline WT & $87.81 \pm 0.7 \mathrm{a}$ & $92.82 \pm 3.91 \mathrm{a}$ & $8.49 \pm 0.25 \mathrm{a}$ & $123.51 \pm 3.23 \mathrm{a}$ & $93.11 \pm 3.09 \mathrm{a}$ & $25.15 \pm 0.79 \mathrm{a}$ & $21.19 \pm 0.57 \mathrm{a}$ \\
WPB106-cas-1 & $87.01 \pm 1.19 \mathrm{a}$ & $95.01 \pm 2.23 \mathrm{a}$ & $8.43 \pm 0.21 \mathrm{a}$ & $124.01 \pm 4.46 \mathrm{a}$ & $96.45 \pm 1.98 \mathrm{a}$ & $25.05 \pm 0.94 \mathrm{a}$ & $20.72 \pm 0.77 \mathrm{a}$ \\
WPB106-cas-2 & $86.51 \pm 0.71 \mathrm{a}$ & $93.03 \pm 2.86 \mathrm{a}$ & $8.38 \pm 0.23 \mathrm{a}$ & $123.52 \pm 3.05 \mathrm{a}$ & $94.29 \pm 2.58 \mathrm{a}$ & $25.29 \pm 0.61 \mathrm{a}$ & $20.82 \pm 0.64 \mathrm{a}$ \\
\hline
\end{tabular}

The data are measured for five plants per line. Values followed by the same letter (a) are not significantly different $(P<0.05)$ 
agronomic traits under normal field conditions. Our study provides a successful case for improving rice salinity tolerance via the CRISPR/Cas9 technology and thus demonstrated that $O S R R 22$ has promising potential to accelerate the improvement of the salinity tolerance in rice breeding.

Many genes involved with salinity tolerance have been identified in rice, such as OsNAC6, OsPPla, OsTPS1, and OsNAP. Transgenic rice plants overexpressing these genes showed an improved tolerance to high salt stresses. However, transgenic plants generated by gene addition are subjected to rigorous genetically modified management. Breeding strategy using CRISPR/Cas9 technology knockdown of rice transcription factor has been demonstrated to be an alternative approach for genetic improvement of rice and avoiding transgenic issue. According to the reports, there are only a few genes acting as a negative regulator of salt tolerance. Although the DST knockdown mutant could effectively improve salt tolerance, it has a large change in agronomic traits, such as leaf width, the panicle number per plant, and the main panicle length (Huang et al. 2009). OsRR22 could significantly improve salt tolerance but no changes were found in other agronomic traits (Takagi et al. 2015). Through our experiments, improved salt tolerance in the OsRR22 knockout lines free of transgene has been verified. Similarly, there was no alteration in the agronomic traits under normal conditions, which has achieved our breeding goals. Salinity tolerance is usually related to drought tolerance. Interestingly, we also carried out the drought-tolerant identification of WT and two homozygous $\mathrm{T}_{2}$ mutant lines at the seedling stage, and the results showed that there was no difference between WT and mutant lines under drought stress (Fig. S1).

In the present study, the Cas9-OsRR22-gRNAinduced mutagenic frequency of $\mathrm{T}_{0}$ plants was $64.3 \%$ and the homozygous rate of $\mathrm{T}_{0}$ mutant plants was $22.2 \%$, which was similar to previous reported values in rice (Zhang et al. 2014; Wang et al. 2016; Zhou et al. 2016). Among six types of induced mutations in $T_{0}$ plants, single-nucleotide insertions were most frequently detected (up to 44.4\%), which is consistent with a previous report (Zhang et al. 2014). In addition, allele mutations could be successfully transmitted to the next generations. Moreover, we observed new mutations within the $T_{1}$ offspring of $\mathrm{rr}-10$, which are probably due to the continuous modification of WT alleles in Cas9-positive $\mathrm{T}_{1}$ lines. T-DNA-free plants could be found in almost all $T_{1}$ segregation population. These results indicate a very convenient production of T-DNAfree homozygous mutation lines in the $\mathrm{T}_{1}$ generation.

In conventional rice breeding, efforts to breed for salinity tolerance have been attempted. However, these usually required approximately one decade due to the lack of accurate screening techniques, lack of adequate resistance resources, and timeconsuming backcrossing procedure (Hoang et al. 2016). Compared to conventional breeding, the CRISPR/Cas9 technology offers the ability to shorten the breeding period and thus significantly reducing cost (Schaart et al. 2016). For example, this experiment showed that we only required 1 year to improve the salt tolerance of WPB106 via CRISPR/ Cas9 technology. Furthermore, the CRISPR/Cas9 technology is more accurate than conventional breeding, since it only creates mutations in the target gene without changing other genes. However, rarely, negative regulatory genes with the desired function and the requirement for a PAM (-NGG) sequence form limitations of the CRISPR/Cas9 system. Conventional breeding has the advantage to improve the complex trait, while the CRISPR/Cas9 technique has the advantage in the mutagenesis of key genes. Therefore, the present study indicates that combining the CRISPR/Cas9 technique with conventional rice breeding could become a very powerful new tool for crop improvement.

Author contributions $\mathrm{ANZ}$ and $\mathrm{YL}$ are equal contributors, and carried out the experiments and vector construction. TFL, FMW, DYK, and JJT did the transformation. ZHC, FYZ, and SFY did the evaluation of salinity tolerance. JGB, JHW, and XXL helped to evaluate the agronomic traits under normal field conditions. XQY, GLL, and LJL made the overall design of this study. All authors read and approved the final manuscript.

Funding information This research was supported by the National High Technology Research and Development Programs of China (Grant No. 2014AA10A603 and 2014AA10A604), the Bill and Melinda Gates Foundation (Grant No. OPP1130530-4), the Shanghai Sailing Program (Grant No. 16YF1410000), the National Natural Science Foundation of China (Grant No. 31501270), and the key program of Shanghai Prospering Agriculture by Science and Technology (Grant No. 2017-02-08-00-08F00071).

Open Access This article is distributed under the terms of the Creative Commons Attribution 4.0 International License (http:// creativecommons.org/licenses/by/4.0/), which permits unrestricted use, distribution, and reproduction in any medium, provided you give appropriate credit to the original author(s) and the source, 
provide a link to the Creative Commons license, and indicate if changes were made.

Publisher's note Springer Nature remains neutral with regard to jurisdictional claims in published maps and institutional affiliations.

\section{References}

Bimpong IK, Manneh B, Sock M, Diaw F, Amoah NKA, Ismail AM, Gregorio G, Singh RK, Wopereis M (2016) Improving salt tolerance of lowland rice cultivar 'Rassi' through markeraided backcross breeding in West Africa. Plant Sci 242:288299

Bortesi L, Fischer R (2015) The CRISPR/Cas9 system for plant genome editing and beyond. Biotechnol Adv 33(1):41-52

Cai Y, Chen L, Liu X, Sun S, Wu C, Jiang B, Han T, Hou W (2015) CRISPR/Cas9-mediated genome editing in soybean hairy roots. PLoS One 10:e136064. https://doi.org/10.1371 /journal.pone.0136064

Chen X, Wang Y, Lv B, Li J, Luo L, Lu S, Zhang X, Ma H, Ming F (2014) The NAC family transcription factor OsNAP confers abiotic stress response through the ABA pathway. Plant Cell Physiol 55(3):604-619

Dionisio-Sese ML, Tobita S (1998) Antioxidant responses of rice seedlings to salinity stress. Plant Sci 135:1-9

Hoang T, Tran T, Nguyen T, Williams B, Wurm P, Bellairs S, Mundree S (2016) Improvement of salinity stress tolerance in rice: challenges and opportunities. Agronomy 6:54. https://doi.org/10.3390/agronomy6040054

Hu H, You J, Fang Y, Zhu X, Qi Z, Xiong L (2008) Characterization of transcription factor gene SNAC2 conferring cold and salt tolerance in rice. Plant Mol Biol 67:169181

Huang XY, Chao DY, Gao JP, Zhu MZ, Shi M, Lin HX (2009) A previously unknown zinc finger protein, DST, regulates drought and salt tolerance in rice via stomatal aperture control. Genes Dev 23(15):1805-1817

Jing W, Zhang W (2017) Research progress on gene mapping and cloning for salt tolerance and variety improvement for salt tolerance by molecular marker-assisted selection in rice. Chin J Rice Sci 31(2):111-123 (in Chinese with English abstract)

Karthikeyan A, Pandian SK, Ramesh M (2011) Transgenic indica rice $\mathrm{cv}$. ADT43 expressing a 1-pyrroline-5-carboxylate synthetase (P5CS) gene from Vigna aconitifolia demonstrates salt tolerance. Plant Cell Tissue Organ Cult 107(3):383-395

Kumar K, Kumar M, Kim SR, Ryu H, Cho YG (2013) Insights into genomics of salt stress response in rice. Rice 6(1):1

Lang N, Buu BC, Ismail AM (2011) Enhancing and stabilizing the productivity of salt-affected areas by incorporating genes for tolerance of abiotic stresses in rice. Omonrice 18:41-49

Li Z, Liu ZB, Xing A, Moon BP, Koellhoffer JP, Huang L, Ward RT, Clifton E, Falco SC, Cigan AM (2015) Cas9-guide RNA directed genome editing in soybean. Plant Physiol 169:960970

Liang Z, Chen KL, Li TD, Zhang Y, Wang YP, Zhao Q, Liu JX, Zhang HW, Liu CM, Ran YD, Gao CX (2017) Efficient DNA-free genome editing of bread wheat using CRISPR/
Cas9 ribonucleoprotein complexes. Nat Commun 8:14261. https://doi.org/10.1038/ncomms14261

Luo LJ (2010) Breeding for water-saving and drought-resistance rice (WDR) in China. J Exp Bot 61:3509-3517

Lutts S, Kinet JM, Bouharmont J (1995) Changes in plant response to $\mathrm{NaCl}$ during development of rice (Oryza sativa $\mathrm{L}$.) varieties differing in salinity resistance. J Exp Bot 46:1843-1852

Ma X, Zhang Q, Zhu Q, Liu W, Chen Y, Qiu R, Wang B, Yang Z, Li H, Lin Y, Xie Y, Shen R, Chen S, Wang Z, Chen Y, Guo J, Chen L, Zhao X, Dong Z, Liu YG (2015a) A robust CRISPR/ Cas9 system for convenient, high-efficiency multiplex genome editing in monocot and dicot plants. Mol Plant 8(8): $1274-1284$

Ma X, Chen L, Zhu Q, Chen Y, Liu YG (2015b) Rapid decoding of sequence-specific nuclease-induced hetero-zygous and biallelic mutations by direct sequencing of PCR products. Mol Plant 8(8):1285-1287

Mahajan S, Tuteja N (2005) Cold, salinity and drought stresses: an overview. Arch Biochem Biophys 444(2):139-158

Munns R, Tester M (2008) Mechanisms of salinity tolerance. Annu Rev Plant Biol 59:651-681

Nieves-Cordones M, Mohamed S, Tanoi K, Kobayashi NI, Takagi K, Vernet A, Guiderdoni E, Périn C, Sentenac H, Véry A-A (2017) Production of low-Cs ${ }^{+}$rice plants by inactivation of the $\mathrm{K}^{+}$transporter OsHAK1 with the CRISPR-Cas system. Plant J 92:43-56

Nishimura A, Aichi I, Matsuoka M (2006) A protocol for Agrobacterium-mediated transformation in rice. Nat Protoc 1(6):2796-2802

Ray DK, Mueller ND, West PC, Foley JA (2013) Yield trends are insufficient to double global crop production by 2050 . PLoS One 8(6):e66428. https://doi.org/10.1371/journal. pone. 0066428

Ren Z, Gao J, Li L, Cai X, Huang W, Chao D, Zhu M, Wang Z, Luan S, Lin H (2005) A rice quantitative trait locus for salt tolerance encodes a sodium transporter. Nat Genet 37(10): $1141-1146$

Schaart JG, van de Wiel CCM, Lotz LAP, Smulders MJM (2016) Opportunities for products of new plant breeding techniques. Trends Plant Sci 21:438-449

SES (standard evaluation system for rice), International Rice Research Institute, Philippines 2002, pp 1-56

Shan Q, Wang Y, Li J, Zhang Y, Chen K, Liang Z, Zhang K, Liu J, Xi JJ, Qiu JL, Gao C (2013) Targeted genome modification of crop plants using a CRISPR-Cas system. Nat Biotech 31(8):686-688

Shao GN, Xie LH, Jiao GA, Wei XJ, Sheng ZH, Tang S, Hu PS (2017) CRISPR/CAS9-mediated editing of the fragrant gene Badh2 in rice. Chin J Rice Sci 31(2):216-222 (in Chinese with English abstract)

Sun S, Chao D, Li X, Shi M, Gao J, Zhu M, Yang H, Luan S, Lin H (2009) OsHAL3 mediates a new pathway in the lightregulated growth of rice. Nat Cell Biol 11:845-851

Svitashev S, Schwattz C, Lenderts B, Young JK, Cigan AM (2016) Genome editing in maize directed by CRISPR-Cas9 ribonucleoprotein complexes. Nat Commun 7:13274. https://doi.org/10.1038/ncomms13274

Takagi H, Tamiru M, Abe A, Kentaro Yoshida K, Uemura A, Yaegashi H, Obara T, Oikawa K, Hiroe Utsushi H, Eiko Kanzaki E, Mitsuoka C, Natsume S, Kosugi S, Kanzaki H, Matsumura H, Urasaki N, Kamoun S, Terauchi R (2015) 
MutMap accelerates breeding of a salt-tolerant rice cultivar. Nat Biotechnol 33(5):445-449

Wang Y, Cheng X, Shan Q, Zhang Y, Liu J, Gao C (2014) Simultaneous editing of three homoeoalleles in hexaploid bread wheat confers heritable resistance to powdery mildew. Nat Biotechnol 32(9):947-951

Wang F, Wang C, Liu P, Lei C, Hao W, Gao Y, Liu YG, Zhao K (2016) Enhanced rice blast resistance by CRISPR/Cas9targeted mutagenesis of the ERF transcription factor gene OsERF922. PLoS One 11:e0154027

Xia H, Huang WX, Xiong J, Yan SG, Tao T, Li JJ, Wu JH, Luo LJ (2017) Differentially methylated epiloci generated from numerous genotypes of contrasting tolerances are associated with osmotic-tolerance in rice seedlings. Front Plant Sci 8: 11. https://doi.org/10.3389/fpls.2017.00011
Zhang H, Zhang J, Wei P, Zhang B, Gou F, Feng Z, Mao Y, Yang L, Zhang H, Xu N, Zhu JK (2014) The CRISPR/Cas9 system produces specific and homozygous targeted gene editing in rice in one generation. Plant Biotechnol J 12(6):797-807

Zhou H, He M, Li J, Chen L, Huang Z, Zheng S, Zhu L, Ni E, Jiang D, Zhao B, Zhuang C (2016) Development of commercial thermo-sensitive genic male sterile rice accelerates hybrid rice breeding using the CRISPR/Cas9-mediated TMS5 editing system. Sci Rep 6:37395. https://doi. org/10.1038/srep37395

Zhu J, Song N, Sun S, Yang W, Zhao H, Song W, Lai J (2016) Efficiency and inheritance of targeted mutagenesis in maize using CRISPR-Cas9. J Genet Genomics 43(1):25-36 